\title{
Procedural learning and associative memory mechanisms contribute to contextual cueing: Evidence from fMRI and eye-tracking
}

\author{
Anna Manelis ${ }^{1,2,3}$ and Lynne M. Reder ${ }^{1,2}$ \\ ${ }^{1}$ Department of Psychology, Carnegie Mellon University, Pittsburgh, Pennsylvania 15213, USA; ${ }^{2}$ The Center for the Neural Basis of \\ Cognition, Carnegie Mellon University, Pittsburgh, Pennsylvania 15213, USA
}

\begin{abstract}
Using a combination of eye tracking and fMRI in a contextual cueing task, we explored the mechanisms underlying the facilitation of visual search for repeated spatial configurations. When configurations of distractors were repeated, greater activation in the right hippocampus corresponded to greater reductions in the number of saccades to locate the target. A psychophysiological interactions analysis for repeated configurations revealed that a strong functional connectivity between this area in the right hippocampus and the left superior parietal lobule early in learning was significantly reduced toward the end of the task. Practice related changes (which we call "procedural learning") in activation in temporo-occipital and parietal brain regions depended on whether or not spatial context was repeated. We conclude that context repetition facilitates visual search through chunk formation that reduces the number of effective distractors that have to be processed during the search. Context repetition influences procedural learning in a way that allows for continuous and effective chunk updating.
\end{abstract}

Numerous studies have provided insights into how people locate a specific object in a complex visual environment (e.g., Treisman and Gelade 1980; Wolfe et al. 1989). An influential paradigm by Chun and Jiang (1998) has added to our understanding by demonstrating the contextual cueing effect. Contextual cueing refers to the facilitation of visual search when a spatial configuration of distractors (for a given target location) in the display is held constant across repetitions compared to when the spatial configurations vary (e.g., Chun and Jiang 1998, 1999). Contextual cueing has been demonstrated in numerous behavioral studies that measure response time differences (e.g., Chun and Jiang 1999; Jiang and Chun 2001; Olson and Chun 2002; Lleras and Von Mühlenen 2004; Bennett et al. 2009) and also in studies that measure eye tracking (e.g., Peterson and Kramer 2001; Tseng and Li 2004; Manginelli and Pollmann 2009). These studies demonstrated that visual search requires less time and fewer eye fixations when the target is embedded in a repeated configuration compared to when it is embedded in a novel configuration. Furthermore, faster search RT corresponds to a fewer number of eye fixations, suggesting an interdependency between the two measures (Tseng and Li 2004).

There has been some debate concerning the nature of the mechanism involved in the contextual cueing effect in terms of the brain regions engaged in the facilitation of search for repeated displays (Chun and Phelps 1999; Manns and Squire 2001; Park et al. 2004; Greene et al. 2007; Preston and Gabrieli 2008). Some researchers have argued that contextual cueing relies on hippocampus (Chun and Phelps 1999; Greene et al. 2007), the region critical for associative memory. Other researchers propose that contextual cueing relies on functioning of the cortical MTL regions (Manns and Squire 2001; Preston and Gabrieli 2008). Part of the evidence for hippocampal involvement was based on patients with damage to the HPC area that showed no advantage

${ }^{3}$ Corresponding author

E-mail anna.manelis@gmail.com

Article is online at http://www.learnmem.org/cgi/doi/10.1101/Im.025973.112. for repeated displays but still a strong improvement in performance with practice (Chun and Phelps 1999). This pattern was replicated with the drug midazolam that mimicked the results found with patients suffering from anterograde amnesia (Park et al. 2004). Additional evidence for hippocampal involvement came from the fMRI study that showed that faster search times for repeated configurations corresponded to greater activation in HPC (Greene et al. 2007).

The fact that anterograde amnesia interferes with contextual cueing but not with speed-up due to practice is consistent with the view that these two effects-contextual cueing and general practice-are unrelated (e.g., Chun and Phelps 1999; Park et al. 2004). The contextual cueing effect is usually considered an instance of implicit learning of the spatial configurations of a target with its distractors that helps guide the subjects' attention toward the target location (e.g., Chun and Jiang 1998, 2003). Several recent studies, however, have challenged this account by showing that contextual cueing is at least partially explained by the contribution of the processes related to response (Kunar et al. 2007; Schankin and Schubö 2009, 2010; Kunar and Wolfe 2011). For example, in the study of Kunar and Wolfe, subjects showed the contextual cueing effect in the contextual cueing paradigm by judging whether the base of the $\mathrm{T}$ is pointing left or right. Later in the task, when the response changed from judging the T orientation to judging whether the $\mathrm{T}$ is present or absent, the contextual cueing effect disappeared. This result suggests that response selection processes may interfere with contextual cueing. Given that response-related processes are procedural in nature, procedural learning in the contextual cueing task may not be independent from context repetition.

The goals of this study are to provide information relevant to the debate concerning the role of HPC in contextual cueing and to investigate whether the two sources of facilitation in the task, relational learning of context to target and procedural learning, are really independent processes. In an attempt to contribute to this debate and show that HPC may be involved in nondeclarative processes, we conducted a concurrent event-related functional 
magnetic resonance imaging (fMRI) and eye-tracking study using an abbreviated version of a contextual cueing task (Bennett et al. 2009). In this task, subjects searched for a rotated $T$, presented among rotated $\mathrm{L}$ distractors, and indicated the direction of the base of the rotated T (Fig. 1). Like the other versions of this paradigm, half of the spatial configurations of L distractors were repeated and half were novel. Importantly, all target locations were repeated from block to block even for novel configurations, but the orientation (pointing left or right) was randomly determined for each $\mathrm{T}$ in each block of trials. Given that the order of the displays was randomly determined, subjects could not know ahead of time where to find a $\mathrm{T}$ for a given trial.

The inclusion of eye-tracking in the fMRI study allows us to use the number of eye fixations to locate the target in a display as a covariate in the fMRI data analysis. Previous studies have argued that eye-movement patterns can serve as a measure of relational memory (e.g., Ryan et al. 2000, 2007; Hannula and Ranganath 2009; Hannula et al. 2010) and correlate with HPC responses (Hannula and Ranganath 2009). We predict that there will be a decrease in the number of fixations to locate the target from the initial exposures (first six blocks of trials) to later exposures (last six blocks of trials) for repeated configurations. Further, we predict that because there is relational learning for repeated configurations, the decrement in the number of fixations for these trials should correlate with the change in activation in the regions critical for associative memory encoding (HPC, parahippocampal, and/or perirhinal cortices).

Teasing apart the contributions from different cognitive processes is not trivial. Behavioral measures often do not provide means for such analyses. In this study, we try to estimate the effect of context repetition on procedural learning using the following logic. Given that for novel displays any improvement must be due to practice at the task, our index of procedural learning is the difference in changes in neural activation from early (first six blocks of trials) to late (last six blocks of trials) task performance. In other words, we define those regions that show reliable contrasts in neural activation for early vs. late task performance for novel configurations as the procedural learning regions.

If contributions from procedural and relational learning are independent, then the patterns of neural activity in procedural learning regions should be the same for novel and repeated configurations. In contrast, if context repetition modulates procedural learning, activation in the procedural learning regions should change depending on whether the spatial configurations are novel or repeated.

\section{Results}

Less than $2.5 \%$ of all trials were removed from the analyses due to incorrect judgment of the target's rotation or due to the failure to make a response during the allotted $6 \mathrm{sec}$. Following multiple pre-

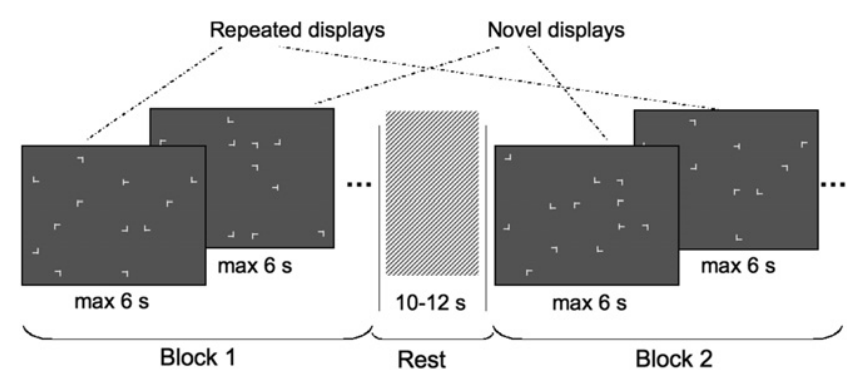

Figure 1. Experimental paradigm. vious studies that used the contextual cueing paradigm (e.g., Chun and Jiang 1998; Bennett et al. 2009), we grouped blocks of trials into epochs in order to increase the power of behavioral and neuroimaging analyses. We restricted our contrasts to the first and last epoch in order to maximize the effect size.

\section{Search RT}

Behavioral data were analyzed using 2 (repeated vs. novel configurations) $\times 2$ (Epoch 1 vs. Epoch 4) repeated measures ANOVA. The behavioral results were largely consistent with previous findings (e.g., Chun and Jiang 1998; Bennett et al. 2009). When the data were analyzed across 13 subjects, there was no main effect of configuration (repeated vs. novel), $P>0.1$, but there was a main effect of epoch, $F_{(1,12)}=12.7, P<0.005$, and an epoch $\times$ configuration interaction, $F_{(1,12)}=6.8, \quad P<0.05$. Search RT decreased during the task for both types of configurations, but the decreases were larger for repeated displays (Fig. 2). These results also held for the subgroup of 11 subjects for whom the eye-tracking data were available. There was no main effect of configuration, $P>0.1$, but there was a main effect of epoch, $F_{(1,10)}=9.6, P<0.05$, and an epoch $\times$ configuration interaction, $F_{(1,10)}=6.4, P<0.05$. Following Chun and Jiang (1998), the contextual cueing effect was calculated as the difference in search RT between the novel and repeated configurations in the second half of the experiment (i.e., collapsed across Epochs 3 and 4). The magnitude of contextual cueing was $121.6 \mathrm{msec}(\mathrm{SE}=50.9)$, which is significantly greater than zero $\left(t_{(12)}=2.4, P<0.05\right)$.

Given that the repetition of configurations did not predict the orientation of the $\mathrm{T}$, it was interesting to compare what we call "stay" vs. "switch" trials. When two successive repetitions across blocks for a given $\mathrm{T}$ (defined by its location in the display) had the same orientation, we call this second presentation a "stay" trial. Conversely, when the orientation for a given $\mathrm{T}$ differed from the preceding block, we call that a "switch" trial. Switch cost was defined as the difference in RT for "switch" vs. "stay" trials for each display type (each T in novel displays also has a fixed location, thus "switch" vs. "stay" can be calculated for novel displays as well). When calculating switch cost, we excluded trials for which subjects made incorrect judgments of the target's orientation (or failed to respond during the allotted six seconds). We also excluded the trials in the next block for which the T's were in the same location as the T's that were incorrectly judged on the previous block.

"Contextual switching" is the term we used to refer to the difference in the switch cost between repeated and novel configurations. The switch cost for novel displays served as a baseline as subjects should not be able to learn a given target's orientation when embedded in novel configurations. To make measures of contextual switching commensurate with contextual cueing, we also calculated the contextual switching effect over the last two epochs in the experiment. The magnitude of contextual switching was only $12 \mathrm{msec}(\mathrm{SE}=57.7)$ and was not different from zero $(P>0.1)$. In spite of this, the correlation between the contextual cueing effect and the contextual switching effect was quite strong, $r=0.6, P<0.05$ (Fig. 3). Subjects showing greater contextual cueing effects (and thus stronger memory representations of the target-context associations) also showed greater switch costs for repeated compared with novel configurations.

\section{Eye tracking}

Eye tracking data were analyzed using 2 (repeated vs. novel configurations) $\times 2$ (Epoch 1 vs. Epoch 4) repeated measures ANOVA. We examined the number of fixations and the latency of the last fixation. 

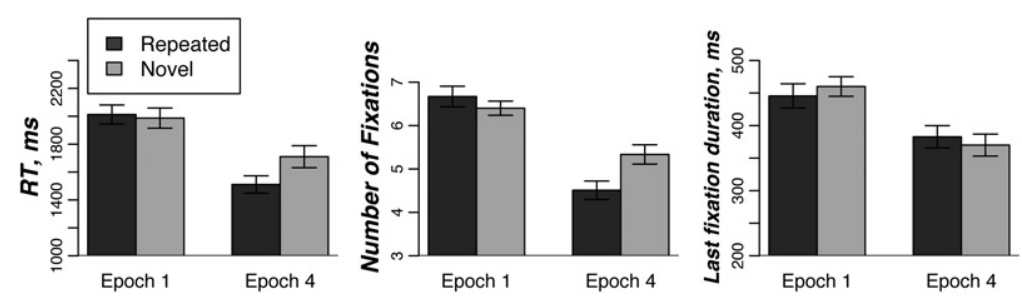

Figure 2. Search RT, number of fixations, and duration of last fixation.

\section{Procedural learning}

Procedural learning was examined by contrasting Epochs 1 and 4 for novel configurations (i.e., N1 vs. N4). Bilateral superior parietal lobule (SPL), right temporo-occipital cortex, right inferior frontal, right middle frontal, and left postcentral gyri decreased activation from N1 to N4 (Table 1). In contrast, the regions in the bilateral posterior cingulate cortex (PCC)/precuneus, the right frontal pole, and the right cerebellum in-

\section{Number of fixations}

There was no main effect of configuration, $P>0.1$, but there was a main effect of epoch, $F_{(1,10)}=41.3, P<0.001$, and an epoch $\times$ configuration interaction, $\left.F_{(1,10)}=8.1, P<0.05\right)$, such that the number of fixations to locate a target decreased from the first (E1) to the last (E4) epoch, but the decreases were greater for repeated than for novel displays (Fig. 2). Consistent with previous studies (e.g., Peterson and Kramer 2001), the mean number of fixations was positively correlated with the mean search RT for both repeated, $r=0.78, P<0.01$, and novel, $r=0.66, P<0.05$, configurations.

\section{Latency of the last fixation}

During the last fixation, subjects located the target, recognized it, selected the response according to the target's orientation and responded. We examined whether the facilitation of search RT for repeated compared with novel configurations can be explained by shorter latencies for the last fixation. While there was a main effect of epoch, $F_{(1,10)}=9.0, P<0.05$, with shorter last fixations in E4 than in E1 (Fig. 2), this effect did not depend on whether the spatial context was repeated $(P>0.1)$.

\section{Neuroimaging}

All neuroimaging results that we report below were thresholded at $P_{\text {corrected }}<0.05$, unless specified otherwise. (For more details, see the Materials and Methods section.)

\section{Correlation in extent of change in number of eye fixations and BOLD signal change during the task}

The hypothesis that practice-related changes in the number of fixations for repeated configurations might be related to the changes in activation of the MTL was tested separately in the right and left HPC, parahippocampal and perirhinal cortices by correlating the E1-E4 changes in that specific MTL region with the E1-E4 changes in the number of eye fixations to locate the target. This analysis revealed that when the spatial context was repeated, the subjects who showed greater reduction from E1 to E4 in the number of saccades to locate the target also showed greater increases in the right HPC from E1 to E4 (z-max $=3.18$ at $[34-24-18]$, 24 voxels, $P_{\text {corrected }}<0.05$ ) (Fig. 4A). The correlation between the change in the BOLD signal extracted from the right HPC and the change in the number of fixations was strong and significant $(r=-0.8$, $P<0.05$ ) for repeated configurations.

As expected, novel configurations did not show a significant correlation between the change in the number of fixations and the change in the BOLD signal in any of the MTL regions, at least at the threshold that was set for this analysis. The correlation between the change in the number of fixations and change in the BOLD signal in the right HPC (the region that was identified in the correlation analysis for repeated displays) was weak and not significant for novel configurations $(r=-0.27, P>0.1)$. creased activation from N1 to N4 (Table 1).

To our surprise, the regions whose activation changed between N1 and N4 did not include basal ganglia. Given that previous research suggested the involvement of basal ganglia in procedural learning (for a review, see Packard and Knowlton 2002) and that the cluster size limit established by AlphaSim was quite high (79 voxels in the cluster), we decided to check whether we would observe the learning-related changes in the basal ganglia if we used a subthreshold cluster size limit (between 70 and 79 voxels in the cluster). This analysis showed that, indeed, right basal ganglia (putamen/caudate nucleus region; $z$-max $=$ 3.25 at [12 20 8], 72 voxels) decreased activation from N1 to N4.

The regions that were revealed in the N1 vs. N4 analysis were used as an ROI mask to investigate (1) procedural learning for repeated configurations (i.e., R1 vs. R4) and (2) the epoch $\times$ configuration interaction (i.e., R1-R4 vs. N1-N4). The results of these analyses are presented in Table 1. Given that basal ganglia activated at the subthreshold level, this region was treated as a separate ROI for the analyses described above. The R1 vs. R4 contrast showed that, like in the N1 vs. N4 contrast, right temporo-occipital cortex (Fig. 5A), bilateral SPL (right SPL is shown on Fig. 5B), and postcentral gyrus had greater activations for R1 than for R4, while right PCC/precuneus and cerebellum had greater activation for R4 than for R1. The R1 vs. R4 analysis in basal ganglia (Fig. 5C) also showed a significant decrease in activation from R1 to R4 in this region ( $z$-max $=3.43$ at [14 2 12], 17 voxels).

The epoch $\times$ configuration interaction analysis in the N1 vs. $\mathrm{N} 4$ mask was conducted to examine whether learning-related changes in brain activation depend on whether the configurations are repeated or novel. A significant interaction effect on brain activation was found in the right inferior temporal (Fig. 5D), the right inferior lateral occipital cortex (LOC)/occipital fusiform regions (Fig. 5E), and the bilateral precuneus (Fig. 5F). The E1 vs. E4 changes were greater for novel compared with repeated

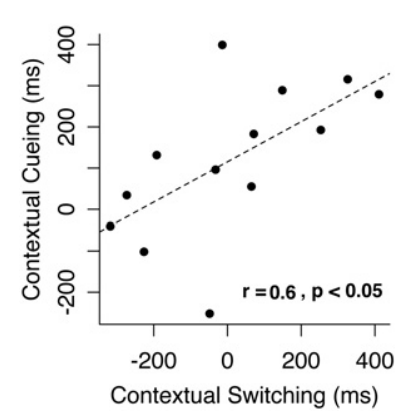

Figure 3. The relationship between the magnitude of the "contextual cueing effect" and the magnitude of the "contextual switching" effect. Greater values of "contextual switching" mean that switching cost (i.e., RT for "switch" trials—RT for "stay" trials) was greater for repeated than for novel configurations. 


\section{Right HPC}
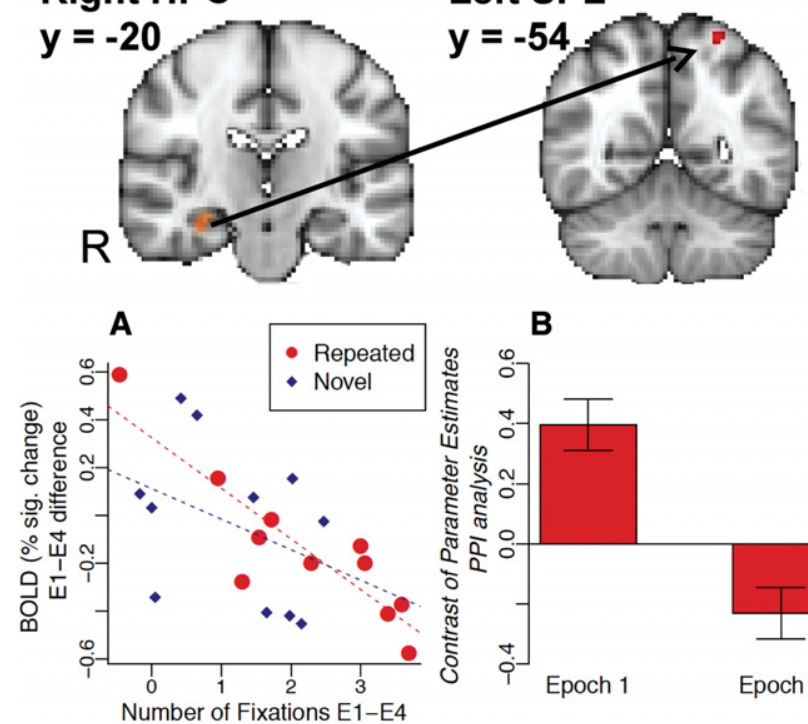

Figure 4. Correlation and psychophysiological interactions analyses. (A) Correlation between the E1-E4 BOLD \% signal change in the right HPC and the E1-E4 NumFix changes for repeated (red) and novel (blue) spatial configurations. Each point on the display represents a subject's data. (B) The right HPC demonstrated decreased functional connectivity with the left SPL for Epoch 4 compared with Epoch 1. This analysis is discussed below (see Results subsection below, Psychophysiological interactions between right hippocampus and procedural learning regions).

configurations in all of these regions (Table 1 ). No epoch $\times$ configuration interaction was found in the right basal ganglia.

\section{Psychophysiological interactions between right hippocampus and procedural learning regions}

We found increased hippocampal involvement for processing of repeated spatial configurations in subjects with greater eye movement facilitation and, arguably, better memory for target-context associations. Therefore, it seemed worthwhile to examine whether learning of repeated configurations over the course of the experiment was related to changes in functional connectivity between this HPC area and the procedural learning regions. We investigated this question using the psychophysiological interactions (PPI) analysis (Friston et al. 1997). The right HPC served as a seed region and the regions revealed in the N1 vs. N4 analysis (described above, in Results subsection Procedural learning) served as target regions. This PPI analysis (Fig. 4B) demonstrated that the functional connectivity between the right HPC and the left SPL decreased significantly from E1 to E4 for repeated spatial configurations $(z=$ 3.4 at $[-24-5664] ; 22$ voxels; $\left.P_{\text {corrected }}<0.05\right)$. Delving further, we conducted PPI analyses separately on E1 and E4 to examine whether the functional connectivity between the right HPC and the left SPL was significantly above a resting baseline during these two epochs. We found a significant positive correlation between right HPC and left SPL for E1 ( $z=2.91$ at $[-24-5666] ; 6$ voxels), but not for E4.

\section{Discussion}

Previously, it had been assumed that the role of HPC in learning and memory was restricted to explicit declarative processes (e.g., Squire 1992). More recently, research has begun to show that the HPC is implicated in implicit learning paradigms such as the contextual cueing task (e.g., Chun and Phelps 1999; Park et al. 2004;
Greene et al. 2007). In response, Manns and Squire (2001) found that when HPC patients did not have damage outside that region, they performed the same as non-amnesic patients. Further, Preston and Gabrieli (2008) reported that HPC is involved only when some explicit memory for spatial configurations is available. However, the evidence is mounting in support of the view that the HPC is not only responsible for relational memory formation (e.g., Davachi 2006; Eichenbaum et al. 2007), but also that this formation need not be explicit (e.g., Reder et al. 2009; Henke 2010; Hannula and Greene 2012).

The current study provides more support for the view that the HPC contributes to performance in relational nondeclarative tasks. The results of our combined fMRI and eye-tracking study have demonstrated that incidental learning of target-context associations in the contextual cueing task relies on the right HPC. The reduction in the number of fixations to locate a target from E1 to E4 was significantly greater for repeated than novel configurations (which may reflect formation of target-context associations). Importantly, subjects with greater reduction in the number of eye fixations from E1 to E4 for repeated configurations showed greater increases in activation in the right HPC from E1 to E4.

These results are consistent with the idea that in the contextual cueing task, the HPC is involved in relational memory for target-context associations (Chun and Phelps 1999; Greene et al. 2007) with eye-movement patterns serving as a measure of relational memory (Ryan et al. 2000, 2007; Hannula and Ranganath 2009). Similarly, these results are consistent with the view that, over repetitions, associations between a target and its surrounding distractors strengthen to form a visual chunk (e.g., Chase and Simon 1973; Gobet et al. 2001). Further, our results are consistent with the findings of Lieberman et al. (2004) that the HPC activates more strongly for chunks that are high in strength compared with chunks that are weaker.

The building of chunks that associate a target location with spatial context would likely involve a change in the functional connectivity between the HPC and the regions supporting the encoding of spatial context. Both HPC and SPL are often involved in encoding and retrieval of spatial information (e.g., Sommer et al. 2005; Piekema et al. 2006; de Rover et al. 2008), with HPC supporting active integration of object and location information (e.g., Manelis et al. 2012). Consistent with the view that subjects were actively forming target-context associations, we found that the right HPC was functionally connected to the left SPL during the initial stage of learning (Epoch 1) for repeated configurations. Later in the task, this connectivity significantly reduced, suggesting that the active stage in the formation of target-context associations is completed and that unitized target-distractor representations have been formed.

The speed of visual search depends on the number of distractors, with faster search for less-populated displays (e.g., Treisman and Gelade 1980; Wolfe et al. 1989). On the view that subjects develop chunks consisting of a target with some of its surrounding distractors, the number of effective distractors in the display diminishes, thereby speeding up search. When the subject's eyes move to that chunk, the target's location within the chunk is reactivated, making access to the target's location easier. This proposal is consistent with findings from Olson and Chun (2002) who found that the contextual cueing effect is due to the portion of the spatial context that is proximal to the target. When the spatial configurations of the distractors around the target (i.e., local context) were repeated from trial to trial, the magnitude of contextual cueing was comparable to that when the spatial configuration of all distractors (i.e., global context) was repeated. Conversely, when the spatial configuration of the proximal distractors was not repeated and only distant configurations of distractors were repeated, no contextual cueing effect was observed. 
Table 1. The results of the neuroimaging data analyses

\begin{tabular}{|c|c|c|c|c|c|c|}
\hline Hemisphere & Region & $\begin{array}{l}\text { Number } \\
\text { of voxels }\end{array}$ & $Z$-max & $x$ & $y$ & $z$ \\
\hline \multicolumn{7}{|c|}{ N1 > N4 (whole brain analysis) } \\
\hline $\mathrm{R}$ & Superior parietal lobule & 648 & 3.86 & 30 & -50 & 70 \\
\hline $\mathrm{R}$ & LOC, inf/occipital fusiform gyrus & 410 & 4.51 & 46 & -72 & -18 \\
\hline $\mathrm{R}$ & Inferior frontal gyrus / precentral g. & 150 & 3.79 & 44 & 10 & 26 \\
\hline $\mathrm{L}$ & Postcentral g. & 105 & 3.47 & -42 & -34 & 60 \\
\hline $\mathrm{R}$ & Frontal pole/middle frontal g. & 105 & 3.18 & 36 & 40 & 36 \\
\hline $\mathrm{R}$ & Middle frontal g. & 98 & 3.25 & 34 & 4 & 58 \\
\hline $\mathrm{L}$ & Superior parietal lobule & 83 & 3.95 & -24 & -54 & 64 \\
\hline \multicolumn{7}{|c|}{ N1 < N4 (whole brain analysis) } \\
\hline B & Cingulate gyrus, post./precuneus & 947 & 3.98 & -4 & -30 & 26 \\
\hline $\mathrm{R}$ & Frontal pole & 101 & 3.45 & 14 & 70 & 22 \\
\hline $\mathrm{R}$ & Cerebellum & 81 & 3.59 & 34 & -70 & -38 \\
\hline $\mathrm{R}$ & Frontal pole & 80 & 3.82 & 22 & 54 & 42 \\
\hline \multicolumn{7}{|c|}{$\mathrm{R} 1>\mathrm{R} 4$ masked by the $\mathrm{N} 1>\mathrm{N} 4$ image } \\
\hline $\mathrm{R}$ & $\begin{array}{l}\text { Postcentral gyrus/superior parietal } \\
\text { lobule }\end{array}$ & 276 & 3.45 & 40 & -36 & 54 \\
\hline $\mathrm{R}$ & Inferior frontal g. & 62 & 3.31 & 46 & 14 & 22 \\
\hline $\mathrm{L}$ & Superior parietal lobule & 38 & 3.67 & -24 & -54 & 60 \\
\hline L & $\begin{array}{l}\text { Postcentral gyrus/superior parietal } \\
\text { lobule }\end{array}$ & 37 & 3.44 & -46 & -36 & 56 \\
\hline $\mathrm{R}$ & $\begin{array}{l}\text { Inferior temporal g./lateral occipital } \\
\text { cortex, infer. }\end{array}$ & 27 & 3.27 & 48 & -60 & -12 \\
\hline \multicolumn{7}{|c|}{$\begin{array}{l}\mathrm{R} 1<\mathrm{R} 4 \text { masked by the N1 }>\mathrm{N} 4 \text { image } \\
\text { none }\end{array}$} \\
\hline \multicolumn{7}{|c|}{$\begin{array}{c}\mathrm{R} 1>\mathrm{R} 4 \text { masked by the } \mathrm{N} 1<\mathrm{N} 4 \text { image } \\
\text { none }\end{array}$} \\
\hline \multicolumn{7}{|c|}{$\mathrm{R} 1<\mathrm{R} 4$ masked by the $\mathrm{N} 1<\mathrm{N} 4$ image } \\
\hline $\mathrm{R}$ & Cingulate gyrus, post./precuneus & 121 & 3.33 & 10 & -48 & 24 \\
\hline $\mathrm{R}$ & Cerebellum & 60 & 4.65 & 36 & -66 & -40 \\
\hline \multicolumn{7}{|c|}{$\mathrm{R} 1-\mathrm{R} 4$ vs. N1-N4 masked by the N1 > N4 image } \\
\hline $\mathrm{R}$ & Inferior temporal g. & 24 & 3.52 & 52 & -58 & -26 \\
\hline $\mathrm{R}$ & LOC, inf/occipital fusiform gyrus & 30 & 3.48 & 46 & -72 & -16 \\
\hline \multicolumn{7}{|c|}{$\mathrm{R} 1-\mathrm{R} 4$ vs. $\mathrm{N} 1-\mathrm{N} 4$ masked by the $\mathrm{N} 1<\mathrm{N} 4$ image } \\
\hline B & Precuneus & 169 & 3.78 & -6 & -60 & 28 \\
\hline
\end{tabular}

(R1) Repeated configurations, Epoch 1; (R4) repeated configurations, Epoch 4; (N1) novel configurations, Epoch 1; (N4) novel configurations, Epoch 4; (g.) gyrus; (post.) posterior; (infer.) inferior.

In this study, we found that the right temporo-occipital cortex (that is involved in object processing; e.g., Chelazzi et al. 1993; Koutstaal et al. 2001; Kristjánsson et al. 2007; Manelis et al. 2011) was differentially sensitive to context repetition. Activation in the superior aspect of this region (Fig. 5A) decreased from E1 to E4 independent of whether context was repeated or not. In the inferior aspect of this region (Fig. 5D,E), however, the repetition of spatial context attenuated the learning-related decreases (the decreases were greater for novel than for repeated configurations). Context repetition also differentially affected activation in the different aspects of the bilateral PCC/precuneus (the region implicated in the processing of visuospatial characteristics of objects) (e.g., Wenderoth et al. 2005; Cavanna and Trimble 2006). While the more anterior aspect of this region showed that activation increases from E1 to E4 independent of context repetition, the more posterior aspect of this region (Fig. 5F) showed greater increases for novel than for repeated configurations.

Given that the procedural aspects of the task were equivalent for both types of display, it might seem surprising that repetition of spatial context modulates activation in regions associated with procedural learning. One reason for such modulation is that, in the repeated display condition, the procedural learning regions may be involved in updating of chunk representations. While the spatial layout of repeated configurations does not change over repetitions, the orientation of the $\mathrm{T}$ is determined randomly and can change from trial to trial. Our analysis suggests that the orientation information is stored with the information about the spatial configuration of distractors. Specifically, subjects with better memory for repeated spatial layouts (and greater contextual cueing effects) also showed larger RT switch costs for repeated compared with novel configurations (see Fig. 3). We believe that storing information about target's orientation helps to facilitate subjects' responses when the $\mathrm{T}$ orientation is repeated from the previous trial, but impairs subjects' performance when the $\mathrm{T}$ orientation is switched. Updating the information about the current orientation of the $\mathrm{T}$ in the chunk representation facilitates subjects' behavioral responses when the T orientation repeats in the next presentation of the same configuration.

The finding that the subjects with the greater contextual cueing effect experience interference when the target orientation changes from the previous block may help us explain the results concerning the duration of the last fixation during visual search. We found that while the duration of this last fixation decreases with practice, these decreases are independent of context repetition. One possibility for the null result is that both target identification and the response to the target are not different for repeated and novel configurations. Another possibility is that subjects spend less time to recognize the target in the repeated than in novel configuration, but it takes more time for them to make a response. The latter idea seems more plausible because first, previous research showed that objects are better recognized in the familiar context (Biederman 1972), suggesting better target identification/recognition for repeated configurations. Second, our study shows that changing the target's orientation from block to block may result in greater switch cost for repeated than for novel configurations (at least in some of the subjects), suggesting slower response selection and production for repeated displays at least on some trials for some of the subjects.

In conclusion, we have demonstrated that facilitation of visual search in repeated spatial configurations relies on the right HPC. In the beginning of learning, the right HPC was strongly connected to the left SPL, suggesting the joint involvement of these regions in formation of target-context associations. At the end of the experiment, we observed no connectivity between these two regions, which may be interpreted as an indication that the target-context associations had already been formed into strong (unitized) chunks. We propose that this chunk formation facilitates visual search by reducing the number of effective distractors that have to be processed during the search.

\section{Materials and Methods}

\section{Participants}

Thirteen right-handed subjects, ages 20-35, from the Psychology Department at Carnegie Mellon University with normal or 


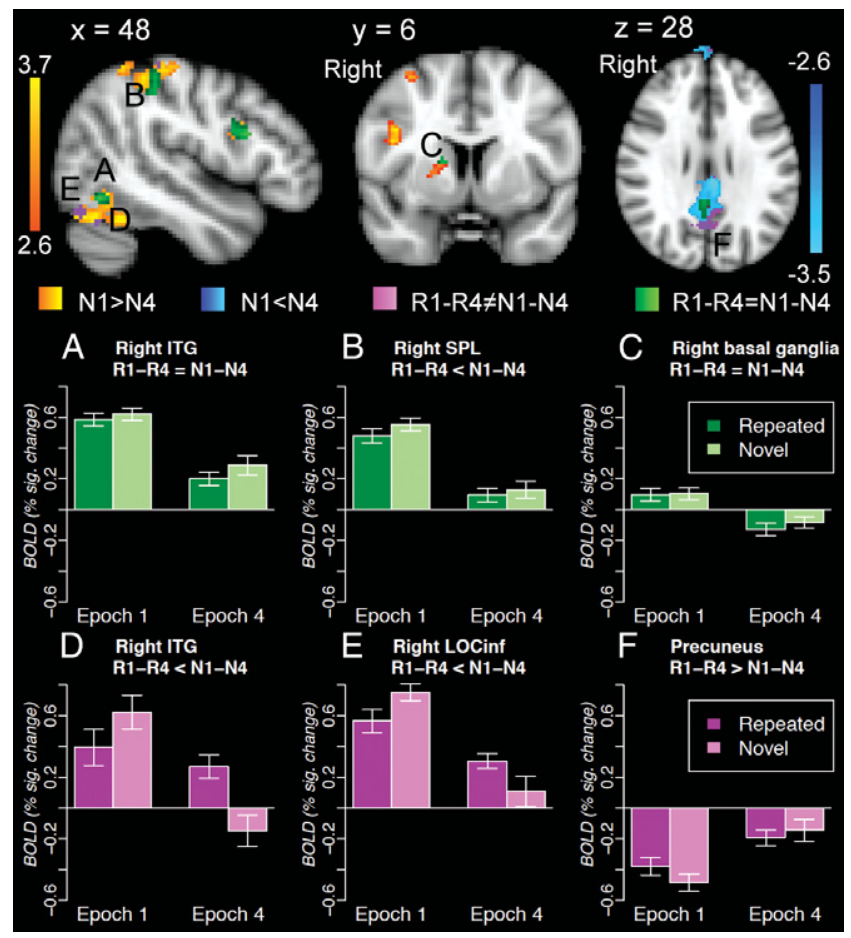

Figure 5. BOLD signal changes over the course of the experiment within procedural (i.e., N1 vs. N4) learning regions. The N1 > N4 contrast is shown in red-yellow. The N1 < N4 contrast is shown in blue. The regions where procedural learning depended on context repetition are shown in magenta. The regions where procedural learning was independent of context repetition are shown in green. The superior aspect of the right inferior temporal gyrus $(A)$, the right $\operatorname{SPL}(B)$, and the right basal ganglia (C), among other regions, showed similar practice-related changes for repeated and novel configurations. The inferior aspect of the right inferior temporal gyrus $(D)$, right inferior lateral occipital cortex (LOCinf) $(E)$, and the posterior aspect of the bilateral precuneus $(F)$ showed greater practice-related changes for novel compared with repeated configurations.

corrected to normal vision participated in this fMRI study. All subjects were treated in accordance with the CMU and Pittsburgh University IRB guidelines. They were compensated $\$ 65$ for their time. The eye-tracking data from two subjects were lost due to eyetracker malfunction. Thus, all analyses involving eye-tracking data are based on the data from 11 subjects.

\section{Stimuli and design}

We used an abbreviated version of a contextual cueing task (Bennett et al. 2009) that consisted of 24 blocks of 12 trials, half repeated and half novel configurations per block. Each spatial configuration consisted of 12 items appearing within the grid of $8 \times 6$ locations. A target was a T rotated $90^{\circ}$ clockwise or counterclockwise, presented among 11 rotated L distractors. Across blocks, the locations of the 12 T's, but not their orientation, were held constant (Fig. 1). Half of these T locations were assigned to have fixed spatial contexts (patterns of L's) and half had a novel context on each trial. This meant that six different repeated configurations were repeated 24 times, while 144 novel configurations were never repeated in the task. The exact patterns of repeated and novel displays as well as their presentation order across blocks were randomly generated for each subject.

Each trial included the presentation of a fixation cross (for 500 $\mathrm{msec}$ ), a display presentation (for a maximum of $6 \mathrm{sec}$ ) and an intertrial interval (ITI) that lasted between 500 and $2500 \mathrm{msec}$. When subjects failed to make a response during the allotted period of time or responded incorrectly concerning target orientation, the trial was removed from the behavioral and neuroimaging data analyses. After each block of 12 trials, there was a 10- to 12 -sec rest period. Stimuli were presented using E-Prime (Psychology Software Tools). Participants indicated the direction of the base of the rotated $\mathrm{T}$ by pressing a button on either the left or right response glove.

\section{Eye tracking}

Eye movements were recorded in the MR scanning environment with a long-range optics eye-tracking system (Model 504LRO, Applied Science Laboratories) that recorded eye position by pupilcorneal reflection obtained by a mirror mounted on the head coil. Nine-point calibrations were performed at the beginning of the scanning session. All eye-tracking data were analyzed off-line using the 5000analysis5_87_03 program that came with the eyetracking software. Eye fixations were analyzed using a maximum change in gaze point of $1^{\circ}$ visual angle and the minimum time of 100 msec.

\section{Image acquisition}

The fMRI data were acquired using a Siemens 3 T Allegra MR system. At the beginning of the experiment, a high-resolution structural image $(\mathrm{TR}=1540 \mathrm{msec}, \mathrm{TE}=3.04 \mathrm{msec}$, slice thickness $=1 \mathrm{~mm}$, $\mathrm{FOV}=205, \mathrm{FA}=8^{\circ}$, number of slices $=192$, resolution $=1 \times 1 \times$ $1 \mathrm{~mm}$ ) was acquired using a magnetization-prepared rapid acquisition in gradient echo (MPRAGE) sequence. A gradient echo, echoplanar sequence $(\mathrm{TR}=2000 \mathrm{msec}, \mathrm{TE}=30 \mathrm{msec}$, slice thickness $=$ $4.0 \mathrm{~mm}, \mathrm{FOV}=205, \mathrm{FA}=79^{\circ}$, number of slices $=35$, resolution $=$ $3.2 \times 3.2 \times 4.0$ ) was used to collect functional data (BOLD signal). Stimuli were presented in a self-paced manner with the constraint that a trial length would not last more than $6 \mathrm{sec}$. This resulted in a variable number of volumes in the subjects' fMRI data (ranging from 710 to 884 volumes). The slices were collected in the ACPC plane with the 14th slice located on the AC-PC.

\section{fMRI data analysis}

All images were processed and analyzed with FSL 4.1.5 software (FMRIB's Software Library, www.fmrib.ox.ac.uk/fsl). For each raw BOLD data set, we applied nonlinear noise reduction (SUSAN, Smallest Univalue Segment Assimilating Nucleus); motion correction (MCFLIRT) (Jenkinson et al. 2002); slice-timing correction using Fourier-space time-series phase-shifting; nonbrain removal using BET (Smith 2002); spatial smoothing using a Gaussian kernel of FWHM $6 \mathrm{~mm}$; and multiplicative mean intensity normalization of the volume at each time point and high-pass temporal filtering (Gaussian-weighted least-squares straight line fitting, with sigma $=25.0 \mathrm{sec})$. A hemodynamic response function $(\mathrm{HRF})$ was modeled using a Gamma function. Coregistration was carried out using FLIRT (Jenkinson and Smith 2001; Jenkinson et al. 2002). In order to transform functional data to the MNI space, BOLD images were registered to the high-resolution structural (MPRAGE) images, the high-resolution images were registered to the MNI152_T1_2-mm template, and the two resulting transformations were concatenated and applied to the original BOLD image (http://www.fmrib.ox.ac.uk/fsl/flirt/gui.html). Functional localization was determined using the Harvard-Oxford cortical and subcortical structural probability atlases (http://www.fmrib.ox.ac.uk/ fsl/fslview/atlas.html).

The FEAT (FMRI Expert Analysis Tool) was used for the firstand higher-level analysis. The first-level analysis included the contrasts of Epoch1 (E1) and Epoch 4 (E4) for repeated (R1-R4) and novel (N1-N4) configurations. Importantly, this contrast for novel configurations reflected the brain response to procedural (task) learning, while for repeated configurations, this contrast reflected the joint effect of procedural and relational learning. The higher-level analysis was carried out using ordinary least squares (OLS) mixed effects. The result of the N1 vs. $\mathrm{N} 4$ contrast was used as a region of interest (ROI) mask both for the contrast of R1 vs. R4 and for the contrast between R1$\mathrm{R} 4$ vs. N1-N4. The latter contrast explored the epoch $\times$ display interaction. 
Cluster size limits for corrected threshold $\left(P_{\text {corrected }}<0.05\right)$ were generated by Monte Carlo simulations (1000 iterations) using the AlphaSim program (http://afni.nimh.nih.gov/pub/dist/ doc/manual/275 AlphaSim.pdf) with FWHM $=6 \mathrm{~mm}$ and uncorrected voxel-wise $P$-value $<0.005$. A whole-brain mask was used to identify cluster size limits for the N1 vs. N4 contrast. According to AlphaSim, a cluster size limit for this contrast was 79 voxels. The resulting image of this contrast, thresholded at $P_{\text {corrected }}<0.05$, was used to identify cluster size limits for the R1 vs. R4 contrast and the R1-R4 vs. N1-N4 contrast. According to AlphaSim, a cluster size limit for the mask image $\mathrm{N} 1>\mathrm{N} 4$ was 18 voxels, while a cluster size limit for the mask image N4 $>$ N1 was 15 voxels.

One of the goals of our study was to test the role of different MTL regions in learning contextual associations. For this reason, we conducted a separate higher-level analysis that used the differences in the number of fixations between $\mathrm{E} 1$ and $\mathrm{E} 4$ as a covariate in the GLM model. This analysis used MTL regions (right and left hippocampus, parahippocampal and perirhinal cortices) as ROIs and was conducted separately for novel and repeated displays. All MTL regions were defined according to the Harvard-Oxford probability atlas with the probability of a voxel being in the ROI at or above $30 \%$. Cluster size limits for $P_{\text {corrected }}$ were determined by a Monte Carlo simulation (1000 iterations) using the AlphaSim program with FWHM $=6 \mathrm{~mm}$ and uncorrected voxelwise $P$-value $<0.01$. The MTL regions served as masks. Cluster size limits for these regions were 22 voxels for right and 21 voxels for left hippocampus, 17 voxels for right and 16 voxels for left peririnal cortex, 13 voxels for right and 15 voxels for left parahippocampal cortex. A cluster size limit for a functional ROI in the right putamen/caudate nucleus was 3 voxels.

\section{Psychophysiological interactions (PPI)}

We performed a functional connectivity analysis using the PPI method (Friston et al. 1997). The region in the right HPC (described above, in Results subsection Correlation in extent of change in number of eye fixations and BOLD signal change during the task) served as a seed region. The procedural learning regions (i.e., the result of the N1 vs. N4 contrast) served as target regions. The PPI analysis model involved: (1) two psychological regressors (R1 and R4); (2) one physiological regressor-a mean time course for the right HPC sensitive to the changes in the number of eye fixations over the course of the experiment; and (3) two interaction terms between the physiological and one of the psychological variables (PPI regressor). In each PPI model, trial types that were not part of the psychological regressor (viz., R2, R3, novel displays, and incorrect [or missing] responses) were included as covariates of no interest. The significant clusters were defined using AlphaSim with FWHM $=6 \mathrm{~mm}$, uncorrected voxel-wise $P$-value $<0.005$ and the image of the N1 vs. N4 contrast as a mask. The first PPI analysis contrasted functional connectivity between the right HPC and the procedural learning regions for E1 vs. E4. The second analysis evaluated whether the functional connectivity in the regions identified by the first analysis was significantly above a resting baseline during Epochs 1 and 4.

\section{Acknowledgments}

This work was supported by grants from the National Institute of Mental Health: 5R01MH052808 and T32MH019983. We thank Lisa Storey for help with the study especially the eye-tracking acquisition and analyses.

\section{References}

Bennett IJ, Barnes KA, Howard JH, Howard DV. 2009. An abbreviated implicit spatial context learning task that yields greater learning. Behav Res Methods 41: 391-395.

Biederman I. 1972. Perceiving real-world scenes. Science 77: 77-80.

Cavanna AE, Trimble MR. 2006. The precuneus: A review of its functional anatomy and behavioural correlates. Brain 129: 564-583.

Chase W, Simon H. 1973. Perception in chess. Cogn Psychol 4: 55-81.
Chelazzi L, Miller EK, Duncan J, Desimone R. 1993. A neural basis for visual search in inferior temporal cortex. Nature 363: 345-347.

Chun MM, Jiang Y. 1998. Contextual cueing: Implicit learning and memory of visual context guides spatial attention. Cogn Psychol 36: 28-71.

Chun MM, Jiang Y. 1999. Top-down attentional guidance based on implicit learning of visual covariation. Psychol Sci 10: 360-365.

Chun MM, Jiang Y. 2003. Implicit, long-term spatial contextual memory. J Exp Psychol Learn Mem Cogn 29: 224-234.

Chun MM, Phelps EA. 1999. Memory deficits for implicit contextual information in amnesic subjects with hippocampal damage. Nat Neurosci 2: 844-847.

Davachi L. 2006. Item, context and relational episodic encoding in humans. Curr Opin Neurobiol 16: 693-700.

de Rover M, Petersson KM, van der Werf SP, Cools AR, Berger HJ, Fernández G. 2008. Neural correlates of strategic memory retrieval: Differentiating between spatial-associative and temporal associative strategies. Hum Brain Mapp 29: 1068-1079.

Eichenbaum H, Yonelinas AP, Ranganath C. 2007. The medial temporal lobe and recognition memory. Annu Rev Neurosci 30: 123-152.

Friston KJ, Buechel C, Fink GR, Morris J, Rolls E, Dolan RJ. 1997. Psychophysiological and modulatory interactions in neuroimaging. NeuroImage 6: 218-229.

Gobet F, Lane PC, Croker S, Cheng PCH, Jones G, Oliver I, Pine JM 2001. Chunking mechanisms in human learning. Trends Cogn Sci 5: 236-243.

Greene AJ, Gross WL, Elsinger CL, Rao SM. 2007. Hippocampal differentiation without recognition: An fMRI analysis of the contextual cueing task. Learn Mem 14: 548-553.

Hannula DE, Greene AJ. 2012. The hippocampus reevaluated in unconscious learning and memory: At a tipping point? Front Hum Neurosci 6: 80. doi: 10.3389/fnhum.2012.0080.

Hannula DE, Ranganath C. 2009. The eyes have it: Hippocampal activity predicts expression of memory in eye movements. Neuron 63: 592-599.

Hannula DE, Althoff RR, Warren DE, Riggs L, Cohen NJ, Ryan JD. 2010. Worth a glance: Using eye movements to investigate the cognitive neuroscience of memory. Front Hum Neurosci 4: 166. doi: 10.2289/ fnhum.2010.00166.

Henke K. 2010. A model for memory systems based on processing modes rather than consciousness. Nat Rev Neurosci 11: 523-532.

Jenkinson M, Smith S. 2001. A global optimisation method for robust affine registration of brain images. Med Image Anal 5: 143-156.

Jenkinson M, Bannister P, Brady M, Smith S. 2002. Improved optimisation for the robust and accurate linear registration and motion correction of brain images. NeuroImage 17: 825-841.

Jiang Y, Chun MM. 2001. Selective attention modulates implicit learning. Q J Exp Psychol 54A: 1105-1124.

Koutstaal W, Wagner AD, Rotte M, Maril A, Buckner RL, Schacter DL. 2001. Perceptual specificity in visual object priming: Functional magnetic resonance imaging evidence for a laterality difference in fusiform cortex. Neuropsychologia 39: 184-199.

Kristjánsson A, Vuilleumier P, Schwartz S, Macaluso E, Driver J. 2007. Neural basis for priming of pop-out during visual search revealed with MRI. Cereb Cortex 17: 1612-1624.

Kunar MA, Wolfe JM. 2011. Target absent trials in configural contextual cuing. Atten Percept Psychophys 73: 2077-2091.

Kunar MA, Flusberg S, Horowitz TS, Wolfe JM. 2007. Does contextual cuing guide the deployment of attention? J Exp Psychol Hum Percept Perform 33: $816-828$.

Lieberman MD, Chang GY, Chiao J, Bookheimer SY, Knowlton BJ. 2004. An event-related fMRI study of artificial grammar learning in a balanced chunk strength design. J Cogn Neurosci 16: 427-438.

Lleras A, Von AV. 2004. Spatial context and top-down strategies in visual search. Spat Vis 17: 465-482.

Manelis A, Wheeler ME, Paynter CA, Storey L, Reder LM. 2011. Opposing patterns of neural priming in same-exemplar vs. different-exemplar repetition predict subsequent memory. NeuroImage 55: $763-772$.

Manelis A, Reder LM, Hanson SJ. 2012. Dynamic changes in the medial temporal lobe during incidental learning of object-location associations. Cereb Cortex 22: 828-837.

Manginelli AA, Pollmann S. 2009. Misleading contextual cues: How do they affect visual search? Psychol Res 73: 212-221.

Manns JR, Squire LR. 2001. Perceptual learning, awareness, and the hippocampus. Hippocampus 11: 776-782.

Olson I, Chun MM. 2002. Perceptual constraints on implicit learning of spatial context. Vis $\operatorname{Cogn}$ 9: 273-302.

Packard MG, Knowlton BJ. 2002. Learning and memory functions of the Basal Ganglia. Annu Rev Neurosci 25: 563-593.

Park H, Quinlan J, Thornton E, Reder LM. 2004. The effect of midazolam on visual search: Implications for understanding amnesia. Proc Natl Acad Sci 101: 17879-17883. 
Peterson MS, Kramer AF. 2001. Attentional guidance of the eyes by contextual information and abrupt onsets. Percept Psychophys 63: 1239-1249.

Piekema C, Kessels RPC, Mars RB, Petersson KM, Fernández G. 2006. The right hippocampus participates in short-term memory maintenance of object-location associations. NeuroImage 33: 374-382.

Preston AR, Gabrieli JDE. 2008. Dissociation between explicit memory and configural memory in the human medial temporal lobe. Cereb Cortex 18: $2192-2207$.

Reder LM, Park H, Kieffaber PD. 2009. Memory systems do not divide on consciousness: Reinterpreting memory in terms of activation and binding. Psychol Bull 135: 23-49.

Ryan JD, Althoff RR, Whitlow S, Cohen NJ. 2000. Amnesia is a deficit in relational memory. Psychol Sci 11: 454-461.

Ryan JD, Hannula DE, Cohen NJ. 2007. The obligatory effects of memory on eye movements. Memory 15: 508-525.

Schankin A, Schubö A. 2009. Cognitive processes facilitated by contextual cueing: Evidence from event-related brain potentials. Psychophysiology 46: $668-679$.

Schankin A, Schubö A. 2010. Contextual cueing effects despite spatially cued target locations. Psychophysiology 47: 717-727.
Smith S. 2002. Fast robust automated brain extraction. Hum Brain Mapp 17: $143-155$.

Sommer T, Rose M, Gläscher J, Wolbers T, Buchel C. 2005. Dissociable contributions within the medial temporal lobe to encoding of object-location associations. Learn Mem 12: 343-351.

Squire LR. 1992. Memory and the hippocampus: A synthesis from findings with rats, monkeys, and humans. Psychol Rev 99: 195-231.

Treisman AM, Gelade G. 1980. A feature-integration theory of attention. Cogn Psychol 12: 97-136.

Tseng Y-C, Li C-SR. 2004. Oculomotor correlates of context-guided learning in visual search. Percept Psychophys 66: 1363-1378.

Wenderoth N, Debaere F, Sunaert S, Swinnen S. 2005. The role of anterior cingulate cortex and precuneus in the coordination of motor behaviour. Eur J Neurosci 22: 235-246.

Wolfe JM, Cave KR, Franzel SL. 1989. Guided search: An alternative to the feature integration model for visual search. J Exp Psychol Hum Percept Perform 15: 419-433.

Received February 14, 2012; accepted in revised form August 2, 2012. 


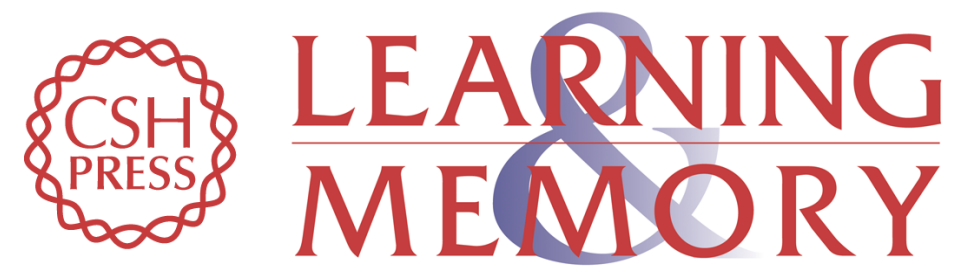

\section{Procedural learning and associative memory mechanisms contribute to contextual cueing: Evidence from fMRI and eye-tracking}

Anna Manelis and Lynne M. Reder

Learn. Mem. 2012, 19:

Access the most recent version at doi:10.1101//m.025973.112

References This article cites 50 articles, 3 of which can be accessed free at: http://learnmem.cshlp.org/content/19/11/527.full.html\#ref-list-1

License

Email Alerting

Receive free email alerts when new articles cite this article - sign up in the box at the Service top right corner of the article or click here. 